\title{
Electron microscopy as a diagnostic tool in pathology
}

\author{
JOHN R. ZOND, DO \\ SCOTT A. COSMI, MS
}

\section{As pathologists begin to exam-} ine increasing numbers of tumors under the electron microscope and the biochemistry begins to characterize molecular change associated with disease states, electron microscopy will gain wider acceptance in clinical practice. The clinician is admonished not to rely on electron microscopy as a sole diagnostic tool, but to use it as an integral part of the available diagnostic armamentarium.

(Key words: Electron microscopy, light microscopy, scanning electron microscopy, transmission electron microscopy, diagnosis, pathology)

During the past 50 years, electron microscopy has evolved from limited use by only a few specialists to a technology widely applied in all fields from material science to pathobiology.

Since the development of the simple microscope by Leeuwenhoek in 1675 , scientists were limited to a useful magnification of about 1000 times by light microscopy, which uses visible light for illumination. The resolution of an optical instrument is approximately half of the wavelength of the image-forming radiation, the limit being about $200 \mathrm{~nm}$.

From the Department of Pathology and Laboratory Medicine, Philadelphia College of Osteopathic Medicine, Philadelphia, $\mathrm{Pa}$, where $\mathrm{Dr}$ Zond is associate professor of pathology, and Mr Cosmi is technical supervisor, section of electron microscopy

Reprint requests to John R. Zond, DO, Philadelphia College of Osteopathic Medicine, 4150 City Ave, Philadelphia, PA 19131-1696.
The wavelength of an electron is nearly 100,000 times smaller than that of light; so, theoretically, the resolution of the electron microscope should be better than light microscopy by that enormous factor. However, as a result of instrument design and image formation difficulties, electron microscopy has achieved only about 1000 times better resolution, a fantastic achievement nonetheless. This increase in resolution provides a useful magnification of nearly 1 million times that is capable of visualizing structures as small as 0.2 $\mathrm{nm}$ in diameter. For comparison, the diameter of a human hair is $50,000 \mathrm{~nm}$.

The conventional transmission electron microscope (TEM) (Fig 1) is by far the electron microscope most widely used by biologists. Although the illuminating radiation of light microscopy and that of electron microscopy differ, the basic lens system and information recording system of these technologies are similar (Fig 2). Whereas the light microscope depends on glass lenses, the electron microscope depends on the electromagnetic lenses for focusing and magnification. In addition, the column of the electron microscope is kept under a high vacuum to prevent interference of air molecules, which would deflect the electrons.

The environmental conditions are also extremely important for achieving quality work with the electron microscope. The room must be free of vibration and stray magnetic fields, which would cause the beam to be unstable. Newer electron microscopes are now userfriendly, having numerous automatic system controls and computer-aided imaging systems. 
Very basic knowledge of the image formation is required for using these newer instruments. The difficulty is not in understanding the instrumentation, but in understanding the image formation recorded by the electron microscope.

\section{Preparing specimens for the electron microscope}

The preparation of specimens is similar for light microscopy and electron microscopy, except the latter technology requires sections to be as thin as 0.05 to $0.1 \mu$, whereas light microscopy requires sections only 1.0 to $100 \mu$ thick. The art of ultramicrotomy has progressed to the point where cutting single cells into hundreds of sections can be done routinely. The use of polymer resins for tissue embedding and freshly cut glass microtome blades has greatly facilitated the achievement of such thin sectioning capability. The stains used in electron microscopy also differ from those used in light microscopy in that they must be electron-dense heavy metals (eg, osmium, lead, and uranium).

The standard method of fixation (aldehydes), dehydration (alcohol), and embedment (epoxy resins) produce an end product that may not represent the in vivo conditions of the tissue. To alleviate much of the artifacts caused by such preparation, new cryofixation and cryoelectron microscopy techniques are now being developed. These cryotechniques provide much improved representative sampling of the in vitro condition because they are 10,000 times faster in immobilizing (stabilizing) tissue components. These cryotechniques are also useful for preparing samples for immunologic studies. Immunologic staining with antibodies conjugated to small gold particles ( 1 to $20 \mathrm{~nm}$ in diameter) promises to dramatically expand the knowledge of the specific sites within the tissue and cells where proteins are made or stored.

\section{Scanning electron microscopy}

Scanning electron microscopes (SEMs) andscanning transmission electron microscopes (STEMs, capable of both TEM and SEM) are also becoming more popular among biologists.

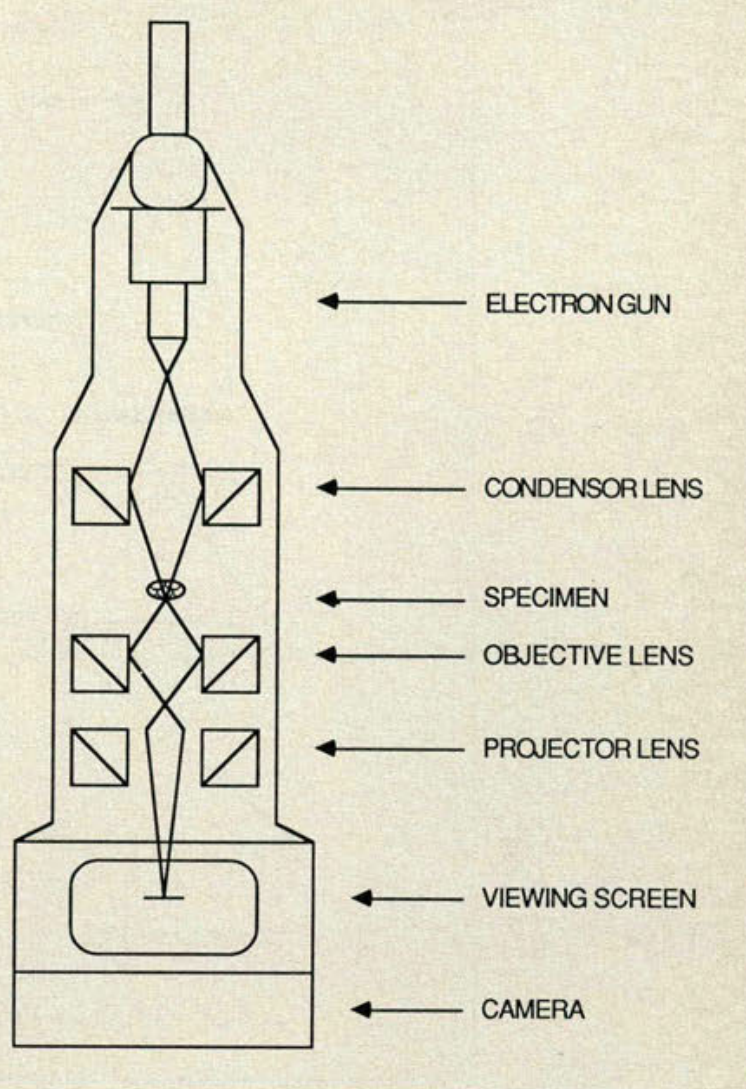

Figure 1. Cross-section schematic of the column of a transmission electron microscope.

As the name implies, the SEM uses a small beam of electrons to scan across the specimen. In the standard SEM mode, a pseudo third dimension is formed (Fig 3) (if a stero pair of a sample are taken, true three-dimensional formations can be recorded).

Scanning electron microscopy is used mainly to visualize surface features, but with accessories such as back-scatter electron and energy-dispersive $\mathrm{x}$-ray detectors, surface or subsurface features may also be visualized. Energy-dispersive $\mathrm{x}$-ray detectors are commonly used to locate asbestos fibers, metals, calcium, and any element heavier than carbon within the tissue and cells. Environmental SEMs have recently been developed that allow hydrated specimens to be visualized. As more innovations are developed in electron microscopy, the information gathered by these highresolution instruments will provide visual evidence to discover or confirm biologic processes 


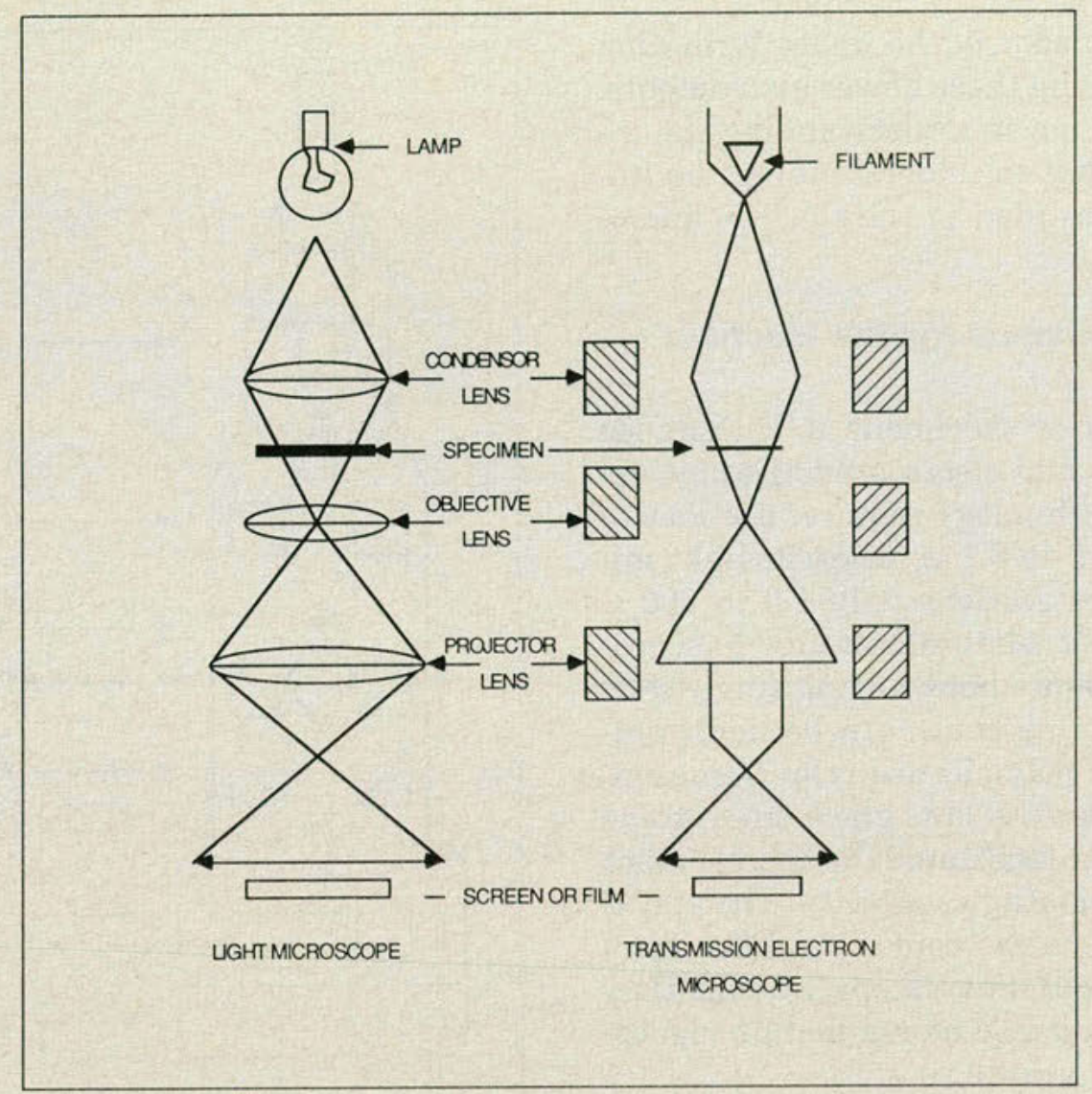

Figure 2. Schematic comparison of light and electron microscopes.

whose existence investigators have only theorized based on previous indirect techniques.

\section{Clinical use of electron microscopy}

Clinicians often have overlooked the diagnostic potential of the electron microscope. Traditionally, the TEM has been used in nephrology for renal biopsies. The evaluation of a patient with a significant proteinuria often begins with a renal biopsy. The TEM is a valuable tool that permits ultrastructural evaluation of the nephron. Subtle alterations, such as thickening of the basement membrane, that are not readily discernible at the light microscopy level are easily recognized with the use of the electron microscope. The structural anomalies of the podocytes and the mesangial and capillary endothelial cells are also more amenable to detection at the electron microscopy level.

Malignant lesions of the kidney can also be evaluated by using electron microscopy. In pe- diatric patients, Wilms' tumor, or neuroblastoma, is characterized by cells with long, narrow cytoplasmic processes, lateral borders abutting each other, microtubules, and dense core granules. In adults, the renal, or clear-cell, carcinoma has intracellularjunctional complexes, lipid droplets, and glycogen granules. Hemangiopericytomas show characteristic intercellular junctions, filaments, and external lamina.

Transmission electron microscopy is also useful in differentiating malignant from benign lesions throughout the body. Tumors of the gastrointestinal tract, respiratory system, reticuloendothelial system, integumentary system, as well as those of soft tissue origin are also amenable to evaluation at the electron microscopy level.

The primary tumor encountered in the gastrointestinal tract is the adenocarcinoma. The diagnostic challenge this tumor presents is the (continued on page 107) 


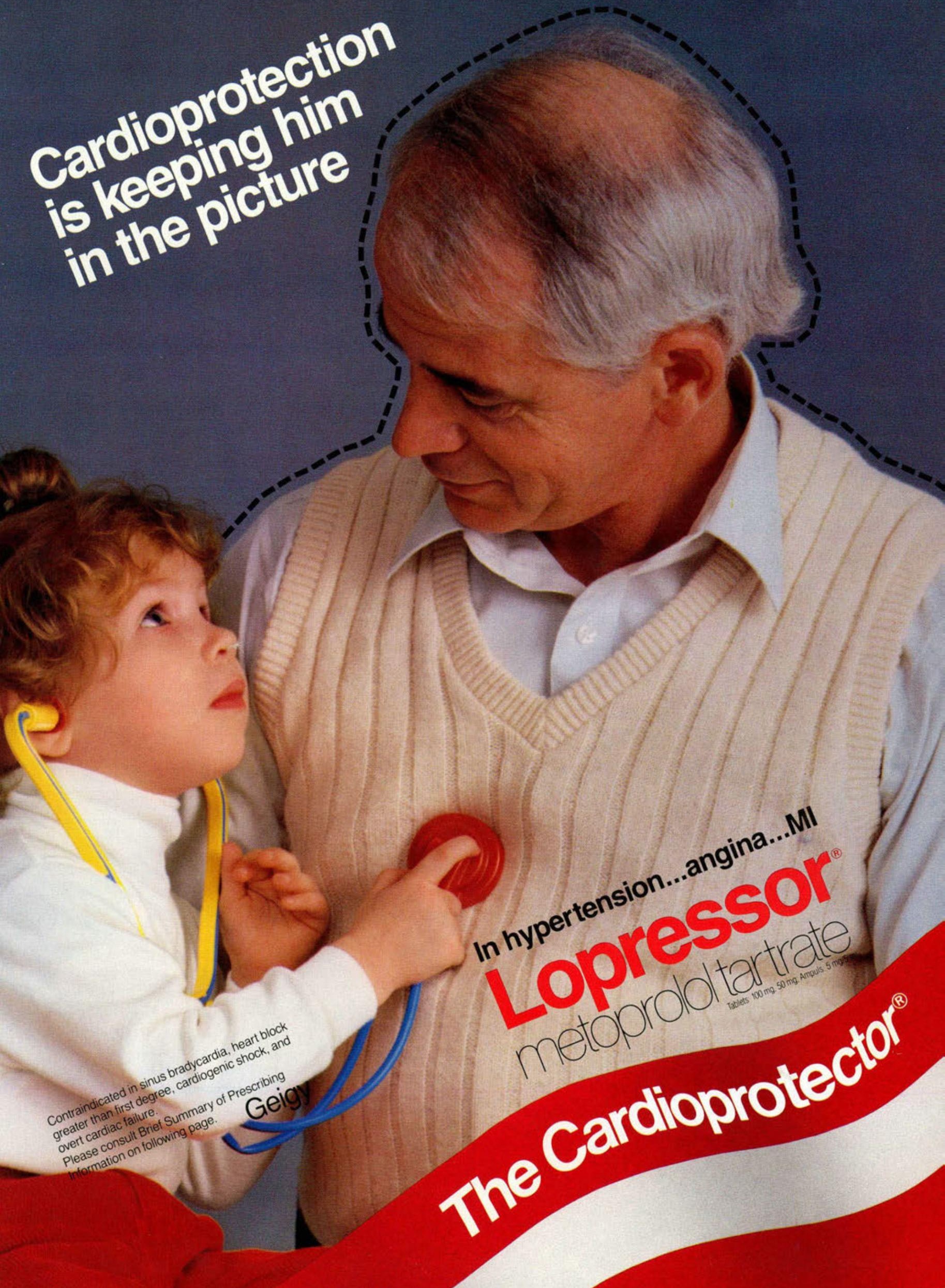




\section{Lopressor $^{8}$}

metoprolol tartrate USP

Tablets

BRIEF SUMMARY (FOR FULL PRESCRIBING

INFORMATION, SEE PACKAGE INSERT)

\section{INDICATIONS AND USAGE}

Hypertension sion. They may be used alone or in combination with other antihypertensive agents.

Angina Pectoris

Lopressor is indicated in the long-term treatment of angina pectoris

\section{Myocardial Infarction}

opressor ampuls and tablets are indicated in the treatment of hemodynamically stable patients with definite or suspected acute myocardialinarction to reduce cardiovascular mortality. reatment with intravenous Lopressor can be initiated as so as the patient's clinical condition allows See DOSAGE AND
ADMINISTRATION, CONTRAINDICATIONS, and WARNINGS Alternatively, treatment can begin within 3 to 10 days of the

\section{CONTRAINDICATIONS}

Hypertension and Angina greater than first degree,

\section{Myocardial Infarction}

copressor is contraindicated in patients with a heart rate $<45$ beats/min; second-and third-degree heart block; significan blood pressure $<100 \mathrm{mmHg}$; or moderate-to-severe cardiac failure (see WARNINGS)

WARNINGS

\section{Hypertension and Angina}

Cardiac Failure: Sympathetic stimulation is a vital component porting circulatory function in congestive heart failure, an beta blockade carries the potential hazard of further depressing myocardial contractility and precipitating more severe failure. nypertensive and angina patients who have congestive hear failure controlled by digitalis and diuretics, Lopressor should be administered cautiously. Both digitalis and Lopressor slow

Patients Without a History of Cardiac Failure: Continued depression of the my a Hardum with beta-blocking agents over the first sign or symptom of impending cardiac failure, patients should be fully digitalized and/or given a diuretic. The response should be observed closely, If cardiac failure continues, de spite adequate digitalization and diuretic therapy. Lopressor should be withdrawn.

Ischemic Heart Disease: Following abrupt cessation of therapy with certain beta-blocking agents, exacerbations of angina pectoris and, in some cases, myocardiall administered Lopressor, particularly in patients with ischemic heart disease, the dosage should be gradually reduced over a period of 1-2 weeks and the patient should be carefully monitored. If angina markedly worsens or acute coronary insufficiency develops, Lopressor administration should be reinstated promptly, at least temporarily, and other measures appropriate for the management of unstable angina should be taken. Patients should be warned against interruption or discontinuation of therapy without the physician's advice. Because coronary artery disease is common and may be unrecognized, it may be prudent not to discontinue Lopressor therapy Bronchospastic Diseases: PATIENTS WITH BRONCHO-
SPASTIC DISEASES SHOULD, IN GENERAL, NOT RECEIVE BETA BLOCKERS. Because of its relative beta, selectivity,

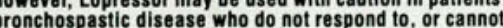
tolerate, other antihypertensive treatment. Since beta selectivity is not absolute, a beta 2 -stimulating agent should be administered concomitantly, and the lowest possible dose of Lopressor should be used. In these circumstances it woul be prudent initially to administer Lopressor in smaller doses three times daily, instead of larger doses two times daily, to avoid the higher plasma levels associated with the longer dosing interval. (SEe DOSAGE AND ADMINISTRATION.)

Major Surgery: The necessity or desirability of withdrawing beta-blocking therapy prior to major surgery is controversial; the impaired ability of the heart to respond to reflex adrenergic gical procedures.

Lopressor, like other beta blockers, is a competitive inhibitor of beta-receptor agonists, and its effects can be reversed by administration of such agents, e.g., dobutamine or iso proterenol. However, such patients may be subject maintaining the heart beat has also been reported with beta

Diabetes and Hypoglycemia: Lopressor should be used with caution in diabetic patients if a beta-blocking agent is required. Beta blockers may mask tachycardia occurring with hypoglycemia, but other manifestations such as dizziness and sweating may not be significantly affected.

Thyrotoxicosis: Beta-adrenergic blockade may mask certain clinical signs (e.g., tachycardia) of hyperthyroidism. Patient suspected of developing thyrotoxicosis should be managed might precipitate a thyroid storm.

Myocardial Infarction

Cardiac Failure: Sympathetic stimulation is a vital component Circulatory function, and beta blockade carries the potential hazard of depressing myocardial contractility and precipitating or exacerbating minimal cardiac failure.

During treatment with Lopressor, the hemodynamic status of the patient should be carefully monitored. If heart failure occurs or persists despite appropriate treatment, Lopressor should be discontinued.
Bradycardia: Lopressor produces a decrease in sinus heart rate in most patients, this decrease is greatest among patients with high initial heart rates and least among patients with low nitial heart rates. Acute myocardial infarction (particularly inferior infarction) may in itself produce significant lowering of the sinus rate. If the sinus rate decreases to $<40$ beats $/ \mathrm{min}$. particularly if associated with evidence of lowered cardiac out. put, atropine $(0.25-0.5 \mathrm{mg})$ should be administered intravenously. Lopressor should be discontinued, and cautious administration of isoproterenol or installation of a cardiac pacemaker should be considered.

AV Block: Lopressor slows AV conduction and may produce significant first- ( $P$-R interval $\geq 0.26 \mathrm{sec}$ ), second-, or thirdheart block

If heart block occurs, Lopressor should be discontinued and atropine $(0.25-0.5 \mathrm{mg})$ should be administered intravenously. If treatment with atropine is not successful, cautious adminisration of isoproterenol or installation of a cardiac pacemaker should be considered

Hypotension: If hypotension (systolic blood pressure $\leq 90$ $\mathrm{mmHg}$ ) occurs, Lopressor should be discontinued, and the hemodynamic status of the patient and the extent of myocardia damage carefully assessed. Invasive monitoring of central venous, pulmonary capillary wedge, and arterial pressures may be required. Appropriate therapy with fluids, positive inotropic agents, balloon counterpulsation, or other treatment modalities bradycardia or AV block, treatment should be directed at reversing these (see above). Bronchospastic Diseases: PATIENTS WITH BRONCHO-
SPASTIC DISEASES SHOULD, IN GENERAL, NOT RECEIVE BETA BLOCKERS. Because of its relative beta, selectivity, Lopressor may be used with extreme caution in patients with Lopressor may be used with extreme caution in patients wi bronchospastic disease. Because it is unknown to what ex
tent beta $a_{2}$-stimulating agents may exacerbate myocardial tent beta ${ }_{2}$-stimulating agents may exacerbate myocardial not be used prophylactically. If bronchospasm not related to not be used prophylactically. If bronchospasm not related to congestive heart failure occurs, Lopressor should be discontinued. A theophylline derivative or a beta ${ }_{2}$ agonist may be administered cautiously, depending on the clinical condition
of the patient. Both theophylline derivatives and beta of the patient. Both theophyline derivatives an
ists may produce serious cardiac arrhythmias. PRECAUTIONS

General

copressor should be used with caution in patients with im paired hepatic function

nformation for Patients

Patients should be advised to take Lopressor regularly and

continuously, as directed, with or immediately following meals.

If a dose should be missed, the patient should take only the

next scheduled dose (without doubling it). Patients should not

discontinue Lopressor without consulting the physician
Patients should be advised (1) to avoid operating auto-

alertness until the patient's response to therapy with Lopressor has been determined; (2) to contact the physician if any difficulty in breathing occurs; (3) to inform the physician or dentis: before any type of surgery that he or she is taking Lopressor. Laboratory Tests

Clinical laboratory findings may include elevated levels of

serum transaminase, alkaline phosphatase, and lactate

dehydrogenase.

Drug Interactions

Cotecholamine-depleting drugs (e.g., reserpine) may have an additive effect when given with beta-blocking agents. Patients treated with Lopressor plus a catecholamine depletor should marked bradycardia, which may produce vertigo, syncope, or postural hypotension.

Risk of Anaphylactic Reaction. While taking beta-blockers, patients with a history of severe anaphylactic reaction to a variety of allergens may be more reactive to repeated cha patients may be unresponsive to the usual doses of epinephrine used to treat allergic reaction.

Carcinogenesis, Mutagenesis, Impairment of Fertility Long-term studies in animals have been conducted to evaluate carcinogenic potential. In a 2-year study in rats at three oral dosage levels of up to $800 \mathrm{mg} / \mathrm{kg}$ per day, there was no increase in the development of spontaneously occurring benign or malignant neoplasms of any type. The only histologic changes that appeared to be orug related were an increased incidence of generally mild focal accumulation of foamy macrophages in pulmonary alveoli and a slight increase in biliary hyperplasia. In a 21-month study in Swiss albino mice at three oral dosage levels of up to $750 \mathrm{mg} / \mathrm{kg}$ per day, benign lung mice (smail adenomas) occurred mon untreated control animals. There was no increase in malignant or total (benign plus malignant) lung tumors, nor in the overall incidence of tumors or malignant tumors. This 21-month study was repeated in $\mathrm{CD}-1$ mice, and no statistically or biologically significant differences were observed between treated and control mice of either sex for any type of tumor.

All mutagenicity tests performed (a dominant lethal study in mice, chromosome studies in somatic cells, a Salmonella

mammalian-microsome mutagenicity test, and a nucleus

anomaly test in somatic interphase nuclei) were negative.

No evidence of impaired fertility due to Lopressor was observed in a study performed in rats at doses

Pregnancy Category $\mathrm{C}$

Lopressor has been shown to increase postimplantation loss and decrease neonatal survival in rats at doses up to 55.5 times the maximum daily human dose of $450 \mathrm{mg}$. Distribution studes in mice confirm exposure ol he retus when Lopressor is administered to the pregnant animal. These studies have There are no adidence of impaired fertility or teratogenicity. There are no adequate and well-controlled studies in pregnan predictive of human response, this drug should be used during pregnancy only if clearly needed.

\section{Nursing Mothers}

Lopressor is excreted in breast milk in very small quantity. An dose of less than 1 liter of breast milk daily would receive a when $L$ epressor is administered

Pediatric Use

Safety and effectiveness in children have not been established. ADVERSE REACTIONS

Hypertension and Angin

ave been mild and transient

Central Nervous System: Tiredness and dizziness have occurred in about 10 of 100 patients. Depression has been reported in about 5 of 100 patients. Mental confusion and short-term memory loss have been reported. Headache, nightCard andinsomnia have also been reported.

Cardiovascular: Shortness of breath and bradycardia have occurred in approximately 3 of 100 patients. Cold extremities; arterial insufficiency, usually of the Raynaud type; palpitations; congestive heart failure; peripheral edema; and hypotension have been reported in about if of 100 patients. (See CON-

Respiratory: Wheezing (bronchospasm) and dyspnea have been reported in about 1 of 100 patients (see WARNINGS). atients. Nausea, dry mouth, gastric pain, constipation, flatulence, and heartburn have been reported in about 1 of 100 patients.

Hypersensitive Reactions: Pruritus or rash have occurred in about 5 o

Miscellaneous: Peyronie's disease has been reported in fewer than 1 of 100,000 patients. Musculoskeletal pain, blurred ision, and tinnitus have also been reported.

There have been rare reports of reversible alopecia

agranulocytosis, and dry eyes. Discontinuation of the drug should be

The oculomucocutaneous syndrome associated with the beta blocker practolol has not been reported with Lopressor.

Myocardial Infarction

Central Nervous System: Tiredness has been reported in about 1 of 100 patients. Vertigo, sleep disturbances, hallucinations, headache, dizziness, visual disturbances, confusion,

Canship is not clear.

Cardiovascular: In the randomized comparison of Lopresso tion, the following adverse reactions were reported

$\begin{array}{lcc}\begin{array}{c}\text { Hypotension } \\ \text { (systolic BP }\end{array} & \begin{array}{c}\text { Lopressor } \\ 27.4 \%\end{array} & \begin{array}{c}\text { Placebo } \\ 23.2 \%\end{array} \\ \begin{array}{c}\text { B } 90 \mathrm{mmHg} \text { ) } \\ \text { Bradycardia } \\ \text { (heart rate }\end{array} & 15.9 \% & 6.7 \% \\ \begin{array}{c}<40 \text { beats } / \mathrm{min} \text { ) } \\ \begin{array}{c}\text { Second- or } \\ \text { third-degree }\end{array}\end{array} & 4.7 \% & 4.7 \% \\ \begin{array}{c}\text { heart block } \\ \text { First-degree } \\ \text { heart block } \\ (P-R \geq 0.26 \mathrm{sec} \text { ) }\end{array} & 5.3 \% & 1.9 \% \\ \begin{array}{c}\text { Heart failure } \\ \text { Respire }\end{array} & 27.5 \% & 29.6 \%\end{array}$

Respiratory: Dyspnea of pulmonary origin has been re-

ported in fewer than 1 of 100 patients.
Gastrointestinal: Nausea and abdominal pain have been

Gastrointestinal: Nausea and abdom

Dermatologic: Rash and worsened psoriasis have been reorted but a drug relationship is not clear

Miscellaneous: Unstable diabetes and claudication have been reported, but a drug relationship is not clear.

(isted above have been reorted with other beta-adrenergic blocking agents and should

Central Nervous System: Reversible mentap ressor.

progressing to catatonia; an acute reversible syndrome charac progressing to catatonia; an acute reversible syndrome char memory loss, emotional lability, slightly clouded sensorium and decreased performance on neuropsychometrics. INDICATIONS

Hematologic: Agranulocytosis, nonthrombocytopenic purpura thrombocytopenic purpura.

Hypersensitive Reactions: Fever combined with aching and sore throat, laryngospasm, and respiratory distress.

OVERDOSAGE

Acute Toxicity

Several cases of overdosage have been reported, some leading

Oral LDso's (mg/kg): mice, 1158-2460; rats, 3090-4670 Signs and Symptoms

Potential signs and symptoms associated with overdosage with Lopressor are bradycardia, hypotension, bronchospasm, and cardiac failure.

There is no specific antidote.

In general, patients with acute or recent myocardial infarction may be more hemodynamically unstable than other pa-
tients and should be treated accordingly (see WARNINGS, Myocardial Infarction).

On the basis of the pharmacologic actions of Lopressor, the ollowing general measures should be employed:

Elimination of the Drug: Gastric lavage should be

Bradycardia: Atropine should be administered. If there is no response to vagal blockade, isoproterenol should be adminis- 

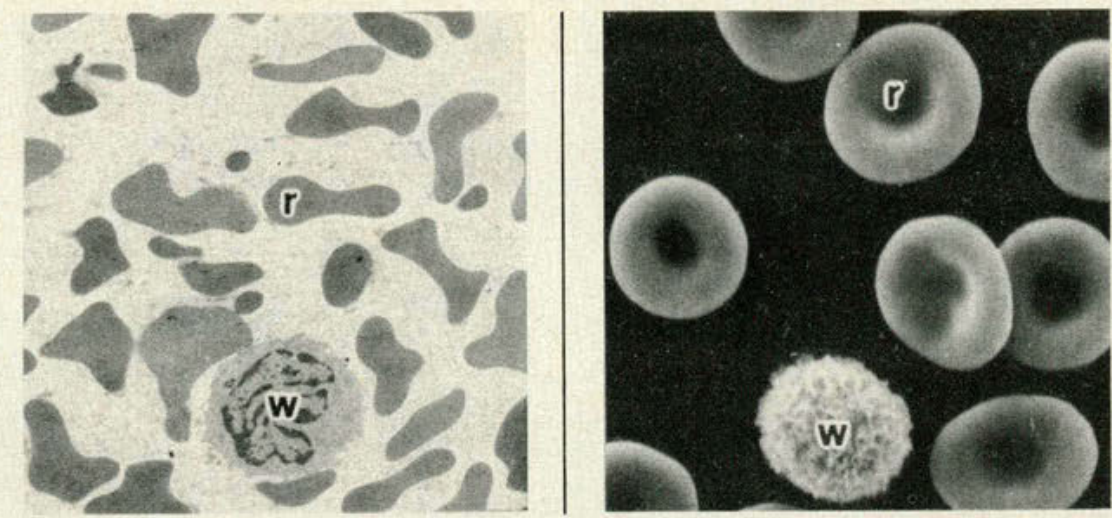

Figure 3. Comparison of transmission electron microscopy scanning (left) and electron microscopy (right) images of red (r) and white (w) blood cells.

determination of the degree of differentiation, information that is of great prognostic significance to the oncologist. Electron microscopy alone, or in combination with immunohisto chemistry, allows the pathologist to evaluate the integrity of the basal lamina, and to more critically compare ultrastructural differences and similarities between normal and malignant cells, such as intercellular junctions and junctional complexes. Immunohistochemistry, when combined with electron microscopy, allows the pathologist to characterize the tumor based on alterations of the cellular biochemistry.

Respiratory tract neoplasms are most commonly of four types: bronchogenic, squamous cell, the primary adenocarcinoma, and metastatic carcinoma. Only the first three will be discussed here. At the electron microscopy level, bronchogenic, or oat-cell, carcinoma is characterized by intracellular junctions and dense core granules in the cytoplasm. Squamous cells characteristically have desmosomes (often difficult to observe at the light microscopy level), tonofibrils (prekeratin filaments), and immunohistochemistry-positive keratohyalin intracellular granules. Adenocarinoma of primary lung origin has an identifiable electron microscopy pattern similar to that of the gastrointestinal tract adenocarcinoma. The neoplasms of the reticulendothelial system that are encountered commonly in electron microscopy are lymphomas, plasmacytomas, and leukemias.

Lymphomas can be categorized as pure histi- ocytic, pure lymphocytic, and mixed histiocyticlymphocytic types. The pure lymphocytic type of lymphomas have a characteristic pattern of heterochromatin along the nuclear membrane. Pure histiocytic lymphoma cells have a cell surface that may be villous or in pseudopods, oval and often indented nucleus, and secondary lysosomes in the cytoplasm.

Multiple myelomas, or plasmacytomas, have a characteristic heterochromatin pattern, indented nucleus, and a cytoplasm rich in endoplasmic reticulum (indicating a great deal of protein production by the cells).

Leukemias are of three basic types: pure lymphocytic, pure monocytic, and mixed myelomonocytic. Lymphocytic leukemias are similar in appearance to lymphocytic lymphomas. Myelocytic leukemic cells have primary azurophilic granules with or without secondary granules, as well as prominent rough endoplasmic reticulum and Golgi apparatuses. Monocytic leukemia cells at the electron microscopy level have a villous surface, folded indented nucleus, prominent Golgi apparatus, and few small primary granules.

Cutaneous neoplasms of chief concern to the pathologist are squamous cell carcinoma and melanoma. The electron microscopy pattern seen in squamous cell carcinoma has been discussed with respect to respiratory tract neoplasms. Melanomas are of two types: amelanotic and melanotic. Melanotic melanomas characteristically have typical melanosomes, whereas amelanotic melanomas have atypical premelanosomes. 


\section{Availability of electron microscopy}

Even though electron microscopy has inestimable diagnostic value, it usually is available only at teaching and research facilities. Most of the facilities are more than willing to cooperate with smaller hospitals. The clinician is advised to consult with the director of the local electron microscopy facility with regard to approriate methods of specimen collection, fixation, storage, and transport. The electron microscopy facility will also request a brief clinical history, which should be provided by the clinician.

\section{Acknowledgment}

Thanks are expressed to Mrs Ollie Margie for her assistance in the preparation of this article.

\section{Bibliography}

Mohanty SB: Electron Microscopy for Biologists. Springfield, Ill, Charles C Thomas Publisher, 1982, chapt 1.

Postek MT, Howard KS, Johnson AH, et al: Scanning Electron Microscopy. Burlington, Vt, Ladd Research Industries, Inc, 1980.

Weakley BS: Biological Transmission Electron Microscopy. New York, NY, Churchill Livingstone, 1981. 